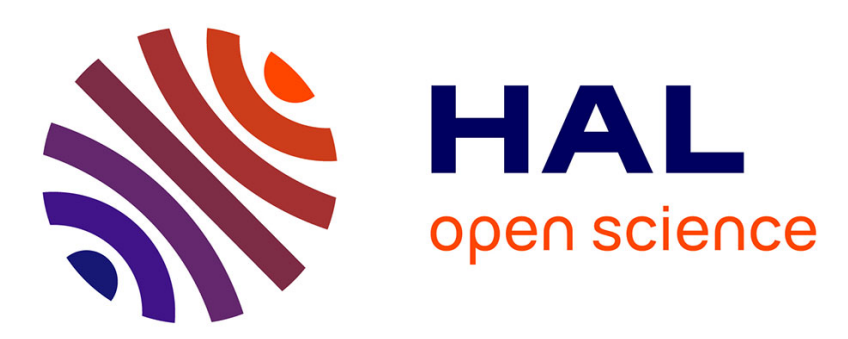

\title{
Electronic structure and carrier dynamics in InAs/InP double-cap quantum dots
}

\author{
Patrice Miska, Jacky Even, Xavier Marie, Olivier Dehaese
}

\section{To cite this version:}

Patrice Miska, Jacky Even, Xavier Marie, Olivier Dehaese. Electronic structure and carrier dynamics in InAs/InP double-cap quantum dots. Applied Physics Letters, 2009, pp.061916. hal-00492421

\section{HAL Id: hal-00492421 \\ https://hal.science/hal-00492421}

Submitted on 15 Jun 2010

HAL is a multi-disciplinary open access archive for the deposit and dissemination of scientific research documents, whether they are published or not. The documents may come from teaching and research institutions in France or abroad, or from public or private research centers.
L'archive ouverte pluridisciplinaire HAL, est destinée au dépôt et à la diffusion de documents scientifiques de niveau recherche, publiés ou non, émanant des établissements d'enseignement et de recherche français ou étrangers, des laboratoires publics ou privés. 


\title{
Electronic structure and carrier dynamics in InAs/InP double-cap quantum dots
}

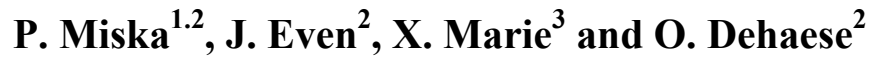 \\ ${ }^{1}$ Institut Jean Lamour \\ CNRS - Nancy-Université - UPV Metz \\ Faculté des Sciences, Boulevard des Aiguillettes \\ BP 239, F-54506 Vandœuvre-lès-Nancy, France
}

${ }^{2}$ Laboratoire FOTON-INSA (UMR-CNRS 6082), 20 Avenue des Buttes de Coesmes, CS 14315, 35043 Rennes Cedex, France

${ }^{3}$ Université de Toulouse, LPCNO ; INSA, CNRS , UPS, 135 Avenue de Rangueil, 31077 Toulouse Cedex 4, France

E-mail : patrice.miska@1pm.u-nancy.fr

PACS numbers : 78.67.Hc, 78.55.Cr, 78.47.Cd, 71.55.Eq, 73.21.La, 71.35.-y, 71.20, 71.48.jc

\begin{abstract}
The carrier dynamics in InAs double-cap quantum dots (DC-QDs) grown on $\operatorname{InP(113)B~are~}$ investigated. The shape of these QDs can be controlled during the growth, yielding an emission wavelength of the system of about $1.55 \mu \mathrm{m}$ at room temperature. The DC-QD dynamics is studied by time-resolved photoluminescence (tr-PL) experiments at low temperature for various excitation densities. A simplified dynamic model is developed, yielding results consistent with experimental data. This analysis yields the determination of the Auger coefficients and the intra-dot relaxation time in this system.
\end{abstract}


Self-organized semiconductor quantum dots (QDs) have been intensively studied in the last years. Their reduced dimensions offer a three-dimensional carrier confinement, yielding discrete atom-like energy spectra. This property is promising for more efficient optoelectronic devices [1,2]. The formation process and the optical properties of many III-V systems such as InAs/GaAs QDs are now well known [2,3]. Many studires have also been devoted to growth and analyzes of optical properties of InAs/InP QDs [4,5] or quantum dashes $\left[{ }^{6}{ }^{7}\right]$.But informations on the carrier dynamics and energy relaxation processes in such InAs/InP QDs are still lacking. Recently, the growth of self-organized InAs QDs on misoriented InP(113)B substrates has been proposed to get QDs with both quantum sizes and a high surface density [8]. In the optimized structures, the control of the maximum QD height of the sample yields the control of their wavelength emission [9]. These structures are named double-cap quantum dots (DC-QDs) and emit at $1.55 \mu \mathrm{m}$ at room temperature [10]. The optical properties of a single DC-QDs layer have been analyzed [10,11,12], and lasing structures were obtained with such nanostructures with low threshold current densities $[13,14]$. In a previous study, we managed to dissociate the two capture (or relaxation) mechanisms described in literature: phonon and Auger assisted relaxations [15]. Nevertheless, a complete dynamic analysis, necessary to give us useful information for the description of the properties of a InAs/InP laser emitting at 1,55 $\mu \mathrm{m}$, was still lacking.

In this paper, we report on the analysis of the dynamic properties of InAs DC-QD layers grown on $\operatorname{InP}(113) \mathrm{B}$ substrate. We used optical experiments together with a dynamic model. The QDs samples are investigated by means of continuous and time-resolved photoluminescence (tr-PL) spectroscopy. We use a static model to describe the energy levels inside the DC-QDs, and a dynamic model based on the resolution of rate equations to fit the tr-PL spectra. The DC-QDs are obtained by the spontaneous Stranski-Krastanow growth 
mode after the deposition of 2.1 InAs monolayers (ML) at $480^{\circ} \mathrm{C}$ on $\operatorname{InP}(311) \mathrm{B}$ substrate with a gas source molecular beam epitaxy system. The double-cap (DC) growth method [10] is then used to control the QD maximum height. The maximum height of the DC-QDs here is of $2 \mathrm{~nm}$ [10], the average DC-QD diameter of about $35 \mathrm{~nm}$ and the surface density of about $10^{10}$ $\mathrm{cm}^{-2}[16]$. Moreover, the WL emission is observed in cw-PL spectra at about $1.05 \mathrm{eV}$ at $10 \mathrm{~K}$ $[16]$.

The cw-PL analysis is performed by exciting the samples at $25 \mathrm{~K}$ with a $647 \mathrm{~nm} \mathrm{Kr}$ laser, and the detection is done by a Peltier cooled PbS detector. Samples are also characterized by time-resolved photoluminescence spectroscopy (tr-PL) at low temperature. The experiments were performed at $10 \mathrm{~K}$ using a $790 \mathrm{~nm}$ Ti-Sapphire laser producing $1.2 \mathrm{ps-}$ long light pulses with a repetition rate of about $82 \mathrm{MHz}$. The tr-PL is then recorded using a synchro-scan streak camera, with an overall time resolution of $\sim 8$ ps [17].

A simple model, described elsewhere [10, 11], and including the DC-QD shape, strain and surface orientation effects is used for the description of the confined electronic states of the DC-QDs. This model gives the probability densities $|\Psi(r, z)|^{2}$, the energies and the oscillator strengths, obtained for a polarisation $\vec{\varepsilon}$ in the electric dipole approximation by :

$$
f_{i \rightarrow f}^{\vec{\varepsilon}}=\frac{2 m_{0}\left(E_{f}-E_{i}\right)}{e^{2} \hbar^{2}}\left|\vec{\varepsilon} \vec{\mu}_{i \rightarrow f}^{\vec{\varepsilon}}\right|
$$

with $E_{f}$ and $E_{i}$ the energies of the final and initial states respectively, and $\vec{\mu}_{i \rightarrow f}^{\vec{\varepsilon}}=\langle f|-e \vec{r}| i\rangle$ the dipole of the transition. Calculated dipole lengths are reported in Fig. 1 as a function of the energy for a truncated lens shaped QD. The model only gives the energies and oscillator strengths of the confined states. The WL propagative states can not be determined (they are represented with a grey area in the spectrum of Figure 1a). The theoretical spectrum is compared with experimental points extracted from tr-PL analysis under high excitation 
density (see ref. 10). The experimental points correspond to the energies of the fundamental $\left(\mathrm{QD}_{0}\right)$ and first excited $\left(\mathrm{QD}_{1}\right)$ states of the DC-QDs, and of two states of the WL visible in the tr-PL spectra. The error bars of the confined energy states correspond to the full width at half maximum of the Gaussian fit of the tr-PL spectra. A reasonable agreement between theoretical and experimental results is obtained despite the simple model used. Former theoretical and experimental studies revealed the electronic structure of $\operatorname{InAs} / \operatorname{InP}(113) \mathrm{B}$ DCQDs $[10,11,12,16]$. For DC-QDs with a maximum height of $2 \mathrm{~nm}$ the transition energy between the first excited states $\left(\mathrm{QD}_{1}\right.$ transition between $1 \mathrm{Pe}$ and $1 \mathrm{Ph}$ states $)$ has been identified around $50 \mathrm{meV}$ above the energy of the fundamental transition $\left(\mathrm{QD}_{0}\right.$ transition between $1 \mathrm{Se}$ and $1 \mathrm{Sh}$ states) [10]. This result has been confirmed by a laser emission obtained with such DC-QDs both at the energy of the fundamental transition and at the energy of the transition between the first DC-QD excited states [13].

After the identification of the confined electronic states, a complete analysis of the carrier dynamics has been done by combining tr-PL experiments and a dynamic model. The different relaxation paths and the corresponding relaxation times we considered are depicted in Fig. 2-a. Based on the experimental results described above, we choose a three-level model (Fig. 1) to model the DC-QD : the WL level where the carriers are created, and two QD confined exciton states $\mathrm{QD}_{0}$ and $\mathrm{QD}_{1}$. We considered the respective spontaneous emission lifetimes of the three levels $\tau_{\text {Spon }}^{W L}, \tau_{\text {Spon }}^{1}$ and $\tau_{\text {Spon }}^{0}$. The values of $\tau_{\text {Spon }}^{W L}$ and $\tau_{\text {Spon }}^{1}$, taken from ref 15 and18 are of 500 and 900 ps respectively ( the fit is not really sensible to the $\tau_{\text {Spon }}^{W L}$ value) . The value of $\tau_{\text {Spon }}^{0}$, taken from ref. 16 is of 1150 ps. For The WL and the first excited state level $\mathrm{QD}_{1}$, we also took into account the capture $\tau_{W L \rightarrow 1}$ and intra-dot relaxation $\tau_{1 \rightarrow 0}$ times and the reverse processes $\tau_{1 \rightarrow W L}$ and $\tau_{0 \rightarrow 1}$ due to the thermal equilibrium of the system. $\tau_{1 \rightarrow W L}$ and $\tau_{0 \rightarrow 1}$ are then calculated as in ref. 19 by applying the detailed balance principle 


$$
\tau_{0 \rightarrow 1}=\tau_{1 \rightarrow 0} \frac{\rho_{0}}{\rho_{1}} e^{\left(\frac{E_{1}-E_{0}}{k_{B} T}\right)} \quad \tau_{1 \rightarrow W L}=\tau_{W L \rightarrow 1} \frac{2 \rho_{1} N_{Q D} V_{Q D} \pi \hbar^{2}}{W L m_{e}^{*} k_{B} T} e^{\left(\frac{E_{W L}-E_{1}}{k_{B} T}\right)}
$$

with $\rho_{0}, \rho_{1}$ and $E_{0}, E_{1}$ the degeneracy and energies of the confined levels $\mathrm{QD}_{0}$ and $\mathrm{QD}_{1}$ respectively $\left(\rho_{0}=1, \rho_{1}=2\right) . E_{0}, E_{1}$ are extracted from Figure 2-a. $N_{Q D}$ is the quantum dot volume density, $V_{Q D}$ the total QD volume inside a square surface with lateral dimension $W$, and $L$ the WL thickness. The considered surface corresponds to the laser spot. Capture $\tau_{W L \rightarrow 1}$ and intra-dot relaxation $\tau_{1 \rightarrow 0}$ times are defined using Auger $\left(C_{W l \rightarrow 1}\right.$ and $\left.C_{1 \rightarrow 0}\right)$ and phonon ( $A_{W L \rightarrow 1}$ and $A_{1 \rightarrow 0}$ ) coefficients as proposed in ref. 19 :

$$
\tau_{W L \rightarrow 1}=\frac{1}{A_{W L \rightarrow 1}+C_{W L \rightarrow 1} N_{W L}} \quad \tau_{1 \rightarrow 0}=\frac{1}{A_{1 \rightarrow 0}+C_{1 \rightarrow 0} N_{W L}}
$$

We assume here a geminate capture process of the electron-hole pair. Moreover, we introduced the occupation rates $f_{0}(t), f_{1}(t), f_{W L}$ of the $\mathrm{QD}_{0}, \mathrm{QD}_{1}$ and WL level respectively. They are defined by :

$$
\begin{array}{lll}
f_{1}(t)=1-P_{1}(t) & f_{0}(t)=1-P_{0}(t) & \\
P_{1}(t)=\frac{N_{1}(t) V_{1 Q D}}{4} & P_{0}(t)=\frac{N_{0}(t) V_{1 Q D}}{2} & f_{W L}=1
\end{array}
$$

with $V_{1 Q D}$ the volume of one quantum dot, and $N_{0}(t)$ and $N_{1}(t)$ the populations of the $\mathrm{QD}_{0}$ and $\mathrm{QD}_{1}$ levels respectively. We consider the $\mathrm{WL}$ as an exciton reservoir. Solving this first set of rate equations with those parameters did not give a good fit of the tr-PL curves. We have evidenced in former study [15] the existence of a direct capture process from the WL onto the $\mathrm{QD}_{0}$ level for all the excitation optical density range. We introduced then in the model a direct relaxation path between the $\mathrm{WL}$ and the $\mathrm{QD}_{0}$ level characterized by a capture time:

$$
\tau_{W L \rightarrow 0}=\tau_{W L \rightarrow 1}
$$


This model yields the resolution of the following rate equations:

$$
\begin{aligned}
& \frac{d N_{W L}}{d t}=\frac{N_{W L}}{\tau_{0}}+\frac{N_{1}}{\tau_{1 \rightarrow W L}} \frac{V_{Q D}}{V_{W L}} f_{C M}-\frac{N_{W L}}{\tau_{W L \rightarrow 1}} f_{1}-\frac{N_{W L}}{\tau_{s p o n}^{W L}}-\frac{N_{W L}}{\tau_{W L \rightarrow 0}} \frac{V_{Q D}}{V_{W L}} f_{0}=0 \\
& \frac{d N_{1}}{d t}=\frac{V_{W L}}{V_{Q D}} \frac{N_{W L}}{\tau_{W L \rightarrow 1}} f_{1}+\frac{N_{0}}{\tau_{0 \rightarrow 1}} f_{1}-\frac{N_{1}}{\tau_{1 \rightarrow W L}} f_{W L}-\frac{N_{1}}{\tau_{s p o n}^{1}}-\frac{N_{1}}{\tau_{1 \rightarrow 0}} f_{0}=0 \\
& \frac{d N_{0}}{d t}=\frac{N_{1}}{\tau_{1 \rightarrow 0}} f_{0}-\frac{N_{0}}{\tau_{0 \rightarrow 1}} f_{1}-\frac{N_{0}}{\tau_{s p o n}^{0}}+\frac{N_{W L}}{\tau_{W L \rightarrow 0}} \frac{V_{Q D}}{V_{W L}} f_{0}=0
\end{aligned}
$$

The number of photogenerated carriers in the WL is given by $N_{W L}(t=0)=\frac{P_{e x c}}{f h v} T^{\text {trans }}\left(1-e^{-\alpha L_{z}}\right)$ with $P_{e x c}$ the average laser excitation power of the tr-PL setup, $h v$ the energy of the incident photons, $f$ the repetition rate of the laser source, $\alpha$ $\left(\sim 10^{4} \mathrm{~cm}^{-1}\right)$ the absorption coefficient in the InP barriers, $L_{z}(30 \mathrm{~nm})$ the thickness of the InP barrier crossed by the photons before reaching the WL, and $T^{\text {trans }}=\frac{4 n_{\text {opt }}^{\operatorname{InP}}}{\left(1+n_{\text {opt }}^{\operatorname{InP}}\right)^{2}}$ the air/InP transmission coefficient evaluated with $n_{\text {opt }}^{\operatorname{InP}}$ (3.9) the optical index of the InP. $\tau_{0}$ is the duration of the laser pulse. With this model the fitting parameters are $A_{W L \rightarrow 1}, C_{W L \rightarrow 1}, A_{1 \rightarrow 0}$, $A_{1 \rightarrow 0}$.

The tr-PL curves obtained from these equations give a good fit of both the experimental rise and the decay times of the $\mathrm{QD}_{0}$ and $\mathrm{QD}_{1}$ levels tr-PL curves in a large excitation intensity range (Fig. 2-b). The rise and decay curves at low excitation density have exponential shape. Under high optical excitation, the $\mathrm{QD}_{1}$ rise and decay curves also have exponential shape, but $\mathrm{QD}_{0}$ decay curve show a plateau. This plateau is due to the saturation of the PL signal corresponding to a complete filling $\mathrm{QD}_{0}$ confined state. Our model gives a good description of this phenomenon. 
Important information for the realization of high frequency lasers is the carrier capture time into the quantum dots. An evaluation of this parameter can be obtained here by evaluating the tr-PL rise time. We have reported in Fig. 3 the experimental and theoretical values of the capture time $\tau_{W L \rightarrow 0}\left(=\tau_{W L \rightarrow 1}\right.$ in our model $)$ as a function of the excitation intensity. We find a good correspondence between the experimental and calculated values. The exciton rise time decreases from 70 ps to 8 ps, and we can identify two regimes: under approximately $50 \mathrm{~W} . \mathrm{cm}^{-2}$ a phonon-assisted regime (where the capture time does not depend on the carrier density), and above $50 \mathrm{~W} . \mathrm{cm}^{-2}$; an Auger regime. Then, the values of phonon $\left(A_{W L \rightarrow 1}\right.$ and $\left.A_{1 \rightarrow 0}\right)$ and Auger $\left(C_{W L \rightarrow 1}\right.$ and $\left.C_{1 \rightarrow 0}\right)$ coefficients of our system were extracted from the fit. We found $A_{W L \rightarrow 1}=1.35 .10^{10} \mathrm{~s}^{-1}, A_{1 \rightarrow 0}=1.10^{10} \mathrm{~s}^{-1}, C_{W L \rightarrow 1}=5.10^{-15} \mathrm{~m}^{3} \mathrm{~s}^{-1}$ and $C_{1 \rightarrow 0}=9.10^{-14} \mathrm{~m}^{3} \mathrm{~s}^{-1}$. These values are close to the ones found in the literature for similar systems [19, 20, 21].

To complete the study, we extracted from the model the intra-dot relaxation time $\tau_{1 \rightarrow 0}$ between the $\mathrm{QD}_{0}$ and $\mathrm{QD}_{1}$ confined states. This time could be measured directly with resonant excitation tr-PL experiment. This calculated intra-dot relaxation time is reported in Fig. 4 as a function of the excitation intensity. The values obtained under high excitation are compatible with the data found in the literature $[22,23]$.

In conclusion, we have analyzed the carrier dynamics in double-cap InAs/InP quantum dots emitting around $1.55 \mu \mathrm{m}$. We identified two confined energy levels, and gave their energies and oscillator strengths. A dynamic study, coupling tr-PL experiments with a simple rate equation model, has revealed the energy relaxation and recombination processes of the exciton. The values of the phonon and Auger coefficients have been deduced and the intra-dot relaxation time between the two confined levels has been estimated. 
1. Self-Assembled InGaAs/GaAs Quantum Dots, edited by Mitsuru Sugawara, Semiconductors and Semimetals vol. 60 (Academic Press, London, 1999).

2. D. Bimberg, M. Grundmann and N. N. Ledentsov, Quantum Dot Heterostructures (Wiley, Chichester, 1998).

3 Self-organized growth on GaAs surfaces, B. A. Joyce and D. V. Vvedensky, Material Science and Engineering R 46, 127 (2004).

4 E. W. Bogaart; R. Nötzel, Q. Gong, J. E. M. Haverkort and J. H. Wolter, Appl. Phys. Lett 86, 173109 (2005).

5 R. Nötzel, S. Anantathanasarn, R. P. J. van Veldhoven, F. W. M. van Otten, T. J. Eijkemans, A.Trampert, B. Satpati, Y. Barbarin, E. A. J. M. Bente, Y.-S. Oei, T. de Vries, E.J.Geluk, B. Smalbrugge, M. K. Smit and J.H. Wolter, Japanese Journal of Applied Physics 45, 6544 (2006).

6 P. Miska, J. Even, C. Platz, B. Salem, T. Benyattou, C. Bru-Chevalier, G. Guillot, Kh.

Moumanis and F. H. Julien, J. Appl. Phys. 95, 1074 (2004)

7 S. Anantathanasarn, R. Nötzel, P. J. van Veldhoven, T. J. Eijkemans, and J. H. Wolter, J. Appl. Phys. 98, 013503 (2005)

8. S. Fréchengues, N. Bertru, V. Drouot, B. Lambert, S. Robinet, S. Loualiche, D. Lacombe and A. Ponchet, Appl. Phys. Lett 74, 3356 (1999).

9. C. Paranthoen, N. Bertru, O. Dehaese, A. Le Corre, S. Loualiche, B. Lambert and G. Patriarche, Appl. Phys. Lett. 78, 1751 (2001).

10. P. Miska, C. Paranthoen, J. Even, O. Dehaese, H. Folliot, N. Bertru, S. Loualiche, M. Senès and X. Marie, Semicond. Sci. Technol. 17, L63 (2002). 
11. P. Miska, C. Paranthoen, J. Even, N. Bertru, A. Le Corre and O. Dehaese, J. Phys.: Condens. Matter 14, 12301 (2002).

12. J. Even, S. Loualiche, P. Miska and C. Platz, J. Phys. : Condens. Matter 15, 8737 (2003).

13. C. Paranthoen, C. Platz, G. Moreau, N. Bertru, O. Dehaese, A. Le Corre, P. Miska, J. Even, H. Folliot, C. Labbé, J.-C. Simon, and S. Loualiche, J. of Cryst. Growth 251, 230 (2003).

14. K. Veselinov, F. Grillot, P. Miska, E. Homeyer, P. Caroff, C. Platz, J. Even, X. Marie, O.

Dehaese, S. Loualiche and A. Ramdane, Opt. Quantum. Electron. 38, 369 (2006).

15. P. Miska, J. Even, O. Dehaese and X. Marie, Appl. Phys. Lett. 92, 191103 (2008).

16. P. Miska, J. Even, C. Paranthoen, O. Dehaese, A. Jbeli, M. Senès and X. Marie, Appl. Phys. Lett. 86, 111905 (2005).

17. M. Sénès, X. Marie, B. Urbaszek, A. Jbeli, P. Renucci, T. Amand and J.-M. Gérard, Phys. Stat. Sol. (C) 1, 594 (2004).

${ }^{18}$ S. Hinooda, S. Loualiche, B. Lambert, N. Bertru, M. Paillard, X. Marie and T. Amand, Appl. Phys. Lett. 78, 3058 (2001).

19. T. W. Berg, S. Bischoff, I. Magnusdottir and J. Mørk, IEEE Photonics Technol. Lett. 13, $541,(2001)$.

20. B. Ohnesorge, M. Albrecht, J. Oshinowo, A. Forchel, Y. Arakawa, Phys. Rev. B 54, $11532(1996)$.

21. A. V. Uskov, McInerney, F. Adler, H. Schweizer and M. H. Pilkuhn, Appl. Phys. Lett. 72, 58 (1998).

22. T. S. Sosnowski, T. B. Norris, H. Jiang, J. Singh, K. Kamath and P. Bhattacharya, Phys. Rev. B 57, R9423 (1998). 
23 E. Péronne, T. Polack, J. F. Lampin, F. Fossard, F. Julien, J. Brault, M. Gendry, O. Marty and A. Alexandrou, Phys. 63, 081307(R) (2001). 


\section{Figure Caption}

Figure $1:$ a) Experimental and b) calculated energies and dipole lengths of the two confined states $\mathrm{QD}_{0}$ and $\mathrm{QD}_{1}$ of the $\mathrm{DC}-\mathrm{QD}$. The error bars of the experimental data correspond to the full width at half maximum of the Gaussian fit of the tr-PL spectra.

Figure 2 : a) Sketch of the radiative and non-radiative mechanisms used to described the exciton dynamics and b) experimental tr-PL curves under high excitation density for the first excited state $\left(\mathrm{QD}_{1}\right.$, circles) and the fundamental state $\left(\mathrm{QD}_{0}\right.$, squares). The fit extracted from the rate equation model is shown with continuous line.

Figure 3 : Experimental rise time $\tau_{W L \rightarrow 0}\left(=\tau_{W L \rightarrow 1}\right.$ in our model $)$ of the fundamental state $\mathrm{QD}_{0}$ as a function of the excitation intensity (full circles). The fit extracted from the rate equation model is shown with continuous line.

Figure 4 : Intra-dot relaxation $\tau_{1 \rightarrow 0}$ between $\mathrm{QD}_{0}$ and $\mathrm{QD}_{1}$ confined states extracted from the dynamic model and reported as a function of the excitation intensity. 


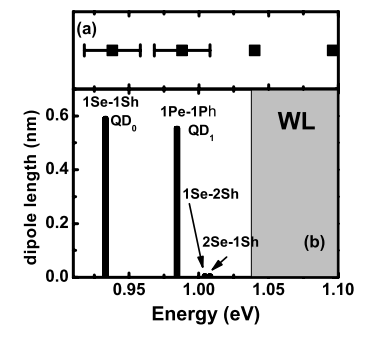



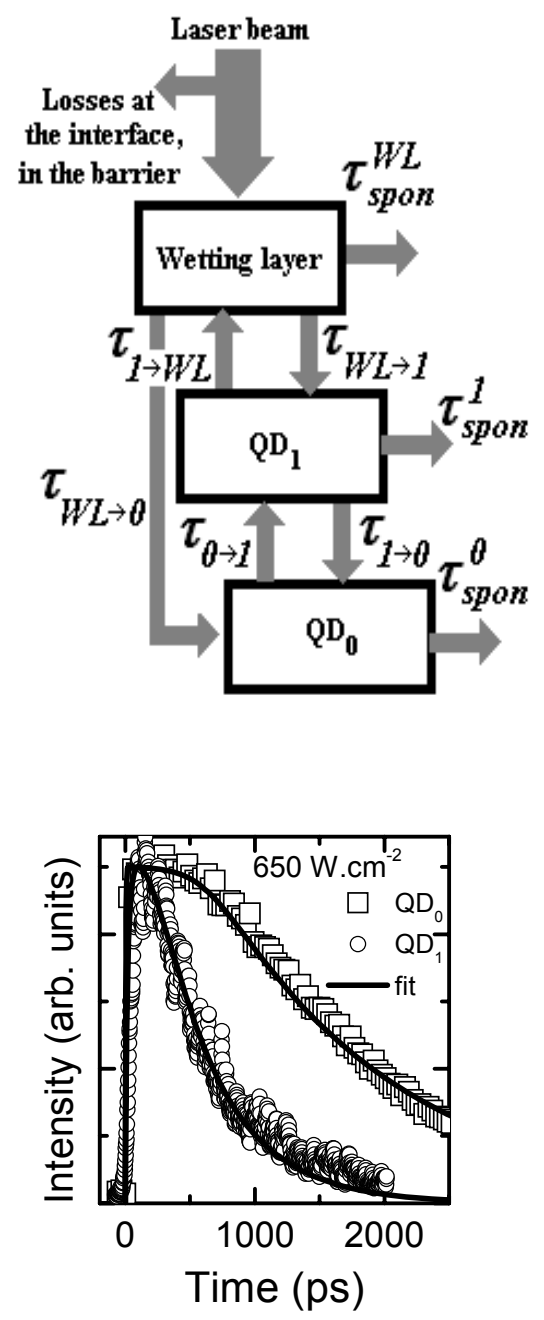


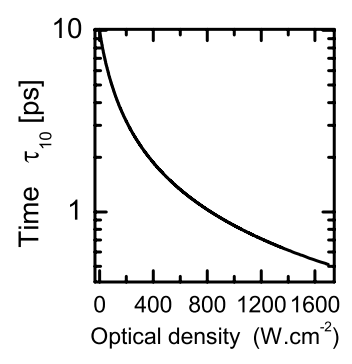

